\title{
L'impact de la crise financière de 2008-2009 sur les grandes universités américaines
}

The Impact of the 2008-2009 Financial Crisis on Top American Research universities

El impacto de la crisis de 2008-2009 en las grandes universidades americanas

\section{Carole Masseys-Bertonèche}

\section{(2) OpenEdition}

Journals

\section{Édition électronique}

URL : https://journals.openedition.org/ideas/648

DOI : 10.4000/ideas.648

ISSN : 1950-5701

\section{Éditeur}

Institut des Amériques

\section{Référence électronique}

Carole Masseys-Bertonèche, «L'impact de la crise financière de 2008-2009 sur les grandes universités américaines », IdeAs [En ligne], 4 | 2013, mis en ligne le 14 octobre 2013, consulté le 20 octobre 2022. URL : http://journals.openedition.org/ideas/648; DOI : https://doi.org/10.4000/ideas 648

Ce document a été généré automatiquement le 20 octobre 2022.

\section{c) (†) $९$}

Creative Commons - Attribution - Pas d'Utilisation Commerciale - Pas de Modification 4.0 International - CC BY-NC-ND 4.0

https://creativecommons.org/licenses/by-nc-nd/4.0/ 


\title{
L'impact de la crise financière de 2008-2009 sur les grandes universités américaines
}

The Impact of the 2008-2009 Financial Crisis on Top American Research universities

El impacto de la crisis de 2008-2009 en las grandes universidades americanas

\author{
Carole Masseys-Bertonèche
}

\section{Introduction}

1 La crise financière de 2008-2009, symbolisée par la chute de la banque Lehman Brothers, a frappé l'ensemble des acteurs de l'économie mondiale. Une étude conduite en décembre 2008 conjointement par The Chronicle of Higher Education et Moody's Investors Service analysait les conséquences probables de cette crise sur les universités américaines et leur prédisait des années très difficiles (Carlson S., 2009). En 2009, ces prévisions pessimistes se confirmaient et les unes après les autres, les grandes universités, privées comme publiques, annonçaient des mesures de restrictions budgétaires importantes.

2 On pouvait alors se demander si, après avoir vu sa puissance financière croître de manière spectaculaire au début des années 2000 grâce à des méthodes de gestion spéculatives qui s'étaient répandues dans toutes les universités, "l'enseignement supérieur américain ne serait pas la prochaine 'bulle' à éclater?", comme le titrait de manière provocante en mai 2009 The Chronicle of Higher Education (Cronin J., Horton H., 2009).

3 Pour répondre à cette question nous essaierons, en premier lieu, d'analyser les changements qu'a subis l'enseignement supérieur américain ces vingt dernières années pour s'adapter et répondre aux crises économiques précédentes. Puis, dans une deuxième partie, nous montrerons comment cette évolution du fonctionnement des 
universités et de leurs modes de financement a accru leur vulnérabilité à la crise financière de 2008-2009. Enfin, nous terminerons cette présentation en tentant d'expliquer les facteurs de résilience des grandes universités américaines aux crises économiques et financières.

\section{Désengagement de l'État et privatisation du système d'enseignement supérieur aux États-Unis : une évolution qui ne date pas de la crise de 2008}

\section{Les causes du désengagement de l'État : choix politiques ou adaptation aux crises économiques?}

4 Le phénomène de privatisation de l'enseignement supérieur aux États-Unis n'a commencé à être étudié que dans les années 2000. Pourtant les mécanismes de la privatisation se sont mis en place dès les années 1980 et se sont accélérés dans les années 1990. Ce mouvement de privatisation de l'enseignement supérieur s'inscrit dans un courant plus large d'évolution de la société américaine et de l'apparition de nouvelles formes de gouvernance tournant autour de quatre thèmes : la réduction des services administratifs, la recherche du résultat, la décentralisation des structures et l'amélioration du service fourni au consommateur. L'idée principale qui sous-tendait ce mouvement était que "le gouvernement n'était pas la solution mais le problème ", comme disait Ronald Reagan, et qu'en obligeant les institutions publiques à se gérer comme des entreprises privées, on améliorerait leur efficacité et on éliminerait le gaspillage (Ehrenberg R., 2006a, 2006b).

Les causes de cette privatisation sont nombreuses; on peut, cependant, citer celles qui ont aidé à façonner le climat menant au désengagement de l'État. La politique de limitation des impôts et des dépenses publiques, qui s'est mise en place à la suite de la révolte fiscale de la fin des années 1970 et qui s'est poursuivie dans les années 1980 et 1990, a été la première cause. Elle a particulièrement touché l'enseignement supérieur (Richburg K., Vick K., 2008). Le transfert de compétences de l'État fédéral aux États fédérés a contribué également à rendre plus difficiles les choix budgétaires à effectuer par les États dans l'allocation de leurs fonds ${ }^{1}$.

6 La mauvaise image des universités auprès de l'opinion publique, à cause d'abord des évènements de 1968 puis des attaques contre « le politiquement correct» en vogue sur les campus à la fin des années 1980, a été un autre facteur qui a certainement joué en leur défaveur dans les choix budgétaires réalisés par les États. L'arrivée au pouvoir des républicains dans de nombreux États semble aussi avoir été une des causes qui a poussé à la privatisation de l'enseignement supérieur (Schmidt P., 2002 : A32 ; Okunade A., 2004 : 123-138). Enfin, et de manière paradoxale, la limitation du nombre de mandats qui s'est répandue à partir des années 1990 dans les législatures de nombreux États a contribué à faire perdre à l'enseignement supérieur un certain nombre de ses "vieux " défenseurs dans l'allocation des fonds ainsi que les avantages d'une politique établie sur le long terme, les législateurs privilégiant les projets réalisables pendant leur mandature (McGuinness A., 1999 ; Hebel S., 2001b ; Leubsdorf B., 2006). 
7 Ce sont donc des choix politiques, faits sous la pression de crises économiques et sociales, qui ont influencé la privatisation des universités américaines. Mais comment peut-on définir ce concept de privatisation de l'enseignement supérieur?

\section{Les caractéristiques de la privatisation : décentralisation, autonomie et concurrence}

8 Le phénomène de privatisation revêt plusieurs caractéristiques. Le désengagement de l'État et la dépendance accrue des universités publiques vis-à-vis de sources de financement privées constituent la première particularité de ce mouvement. L'enseignement supérieur n'a plus été une priorité dans les budgets des États et les différentes périodes de récession qui ont touché les États-Unis depuis la fin des années 1970 ont renforcé le mouvement ${ }^{2}$. Même si cette réduction des financements publics n'a été qu'une baisse relative et n'a pas touché tous les États de la même façon, le déclin de la part des fonds publics dans le budget des universités est un phénomène qui a affecté toutes les institutions d'enseignement supérieur et plus particulièrement les grandes universités de recherche, porte-drapeaux de l'enseignement public aux États-Unis ${ }^{3}$.

9 La deuxième caractéristique du phénomène de privatisation est le mouvement des institutions d'enseignement supérieur vers plus d'autonomie de leur gouvernance à la suite de la diminution du contrôle direct de l'État ${ }^{4}$. Cette décentralisation de la gouvernance s'est mise en place à travers trois approches différentes. La première a consisté à transférer certaines prises de décisions tout en conservant les structures en place. La deuxième, plus radicale, a été la désagrégation des grands multi-campus (Schmidt P., 2001). La troisième s'est effectuée par le changement de statut des universités pour leur donner plus d'autonomie de décision (Hebel S., 2000).

10 Cette décentralisation de l'autorité, commencée dans les années 1980, s'est accompagnée d'un abandon de la fixation des frais de scolarité par l'État au profit des universités. Cette délégation de pouvoir a été suivie d'un encouragement des étudiants à s'autofinancer par des programmes d'emprunts ou de préfinancement de leurs frais de scolarité (Prepaid Tuition and College Savings Plans)5. Le financement des études supérieures est passé d'une responsabilité publique à une responsabilité privée selon le principe cher à Reagan "qu'on devait rendre les étudiants responsables du financement de leur éducation » (Parsons M., 1997 : 62). En parallèle, les bourses ont commencé à être distribuées non plus en fonction de critères de revenus mais de mérite. Il est intéressant de noter que ce sont les États du Sud qui sont les premiers à avoir mis en place ces programmes d'encouragement des étudiants en fonction de leur réussite scolaire. Le premier programme de bourse pour aider les meilleurs élèves, HOPE (Helping Oustanding Pupils Educationally), a été lancé en 1993 en Géorgie et, en 2003, 13 États, la plupart localisés dans le Sud, l'avaient adopté6.

11 Cette popularité croissante des notions de mérite et de compétition s'est aussi manifestée au niveau des institutions car les aides de l'État n'ont plus été versées directement aux universités mais aux étudiants, leur donnant ainsi le choix de leur établissement. Cette décision qui a été prise dans le cadre de la loi sur l'enseignement supérieur de 1972 (Higher Education Act of 1972) marque, pour beaucoup de spécialistes de l'enseignement supérieur, le début du processus de "marchandisation » de l'aide financière aux étudiants. Selon le sénateur Pell, qui a mené la bataille en faveur de cette loi, il convenait de déléguer la prise de décision aux consommateurs de savoir (Gladieux 
L., Wolanin T., 1975). De même, les bourses ont été offertes sans distinction à tous les étudiants quel que soit leur choix d'institutions ce qui n'a fait qu'accroître la concurrence entre les différents secteurs : privé à but non lucratif, privé à but lucratif et public.

Intéressons-nous maintenant aux conséquences de cette marchandisation et de cette compétition accrue sur la gestion des institutions d'enseignement supérieur. Comment ces changements ont-ils accru leur vulnérabilité dans le contexte de la crise actuelle?

\section{L'évolution de la gestion et des modes de financement des universités américaines : une source de vulnérabilité accrue face à la crise}

\section{La marchandisation des universités américaines : la perte de notion de service public encouragée par des États en crise et par la croyance dans le tout marché}

13 La marchandisation ou "commercialisation » des universités américaines, selon le terme utilisé par Derek Bok, ancien président d'Harvard (Bok D., 2003), a revêtu plusieurs formes mais s'est manifestée principalement autour de la compétition acharnée que se livrent les universités publiques et privées pour augmenter leurs sources de financement. Si les modes de gestion des universités divergent en fonction de leur statut, leurs sources de fonds sont les mêmes à l'exception du financement en provenance des États fédérés qui est réservé aux universités publiques. C'est donc dans la répartition des ressources que réside la différence. À la fin des années 1970, les universités publiques étaient financées à presque $70 \%$ par de l'argent public. Le financement en provenance de fonds privés ne dépassait pas les $3 \%$, le reste étant couvert par les droits d'inscription et les ventes de biens et de services ${ }^{7}$. Les universités privées bénéficiaient aussi largement de l'argent des contribuables avec des budgets financés, pour certaines d'entre elles, à $50 \%$ par des financements fédéraux. Cet âge d'or du financement public de l'enseignement supérieur se termina avec les événements de 1968 et la crise pétrolière des années $1970^{8}$.

Les universités privées furent les premières à s'orienter vers une plus grande diversification de leurs ressources financières; démarche qui avait pour but de réduire leur dépendance à l'argent public. Tout d'abord, elles firent le choix d'améliorer la gestion de leur endowment 9 . Accroitre le montant de leur endowment a toujours été une priorité des universités américaines et pour ce faire elles s'inspirent souvent de ce qui se fait à Harvard, l'université qui dispose de l'endowment le plus doté des États-Unis. Dès 1974, Harvard a créé la Harvard Management Company, une société d'investissement dont l'unique mission est de gérer l'endowment de l'université ${ }^{10}$.

Yale a très vite emboîté le pas à Harvard, en organisant aussi une " gestion maison » de son endowment, sous le leadership d'un de ses anciens élèves, David Swensen ${ }^{11}$. Swensen est très vite devenu le gourou de la nouvelle stratégie de gestion des endowments des universités, basée sur des investissements alternatifs plus agressifs, plus risqués mais à forte rentabilité1 ${ }^{12}$. L'équipe qui l'entourait n'était pas nombreuse, mais elle déléguait une partie du travail à de jeunes sociétés d'investissement privées et indépendantes qui avaient la possibilité d'investir dans des valeurs parfois très controversées, ce que ne 
pouvaient pas faire les gestionnaires de l'endowment de Yale sans s'attirer les foudres du monde universitaire déjà très choqué par les salaires et bonus considérables qu'ils touchaient.

Impressionnées par les performances d'Harvard et de $\mathrm{Yale}^{13}$, les grandes universités privées et publiques ont rapidement adopté les mêmes stratégies d'investissement et jusqu'en 2007 toutes les universités d'élite affichaient des taux de croissance spectaculaires de leurs endowments ${ }^{14}$.

17 Cette croissance s'accompagne d'une grande inégalité puisque les 20 institutions les plus riches possèdent plus de la moitié du total des endowments des 4000 institutions d'enseignement supérieur américaines. Parmi ces 20 institutions qui ont des endowments qui dépassent les 4 milliards de dollars figurent cinq universités publiques $^{15}$.

18 En effet, de la même manière que les universités privées n'ont jamais été complètement indépendantes de l'État, les grandes universités publiques ont toujours bénéficié du soutien de la philanthropie ${ }^{16}$. La recherche de fonds privés, qui au départ était limitée à quelques grandes universités publiques, s'est fortement accentuée dans les années 1980 et 1990 pour toutes les institutions avec la diminution des dotations d'État dans leurs budgets. De 1979 à 1984 le pourcentage des institutions publiques se lançant dans des collectes de fonds a doublé (Coldren S., 1982) et cette augmentation n'a fait que se confirmer et s'accélérer dans les années 1990.

Beaucoup de responsables de l'enseignement supérieur public déplorent cet état de fait dans lequel « les universités publiques sont passées d'une phase où l'État les soutenait, à une phase où l'État les aidait, pour en arriver à une phase où l'État ne fait plus que les héberger » (Breneman D., 2002). Cette course à l'argent a gagné toutes les universités. La réussite des collectes de fonds est devenue un objectif prioritaire dans lequel les présidents, les doyens, le personnel administratif, les anciens élèves et même les enseignants et les étudiants se sont complètement investis. Les nouvelles rentrées de fonds, bien qu'ayant facilité la gestion des universités, ont eu aussi pour conséquences d'entraîner des dépenses pas toujours nécessaires et un train de vie parfois au-dessus des moyens des institutions. Des besoins grandissants, réels ou supposés, ont conduit les universités à chercher à accroître leurs autres sources de financement parmi lesquelles les droits d'inscription.

20 Variable d'ajustement, l'accroissement des frais de scolarité a été un moyen pour les universités publiques d'amortir les variations budgétaires dues aux financements souvent erratiques des États mais aussi de contribuer à la course au «toujours plus de revenus ». Stables jusqu'à la fin des années 1970, les droits d'inscription se sont envolés dans les années 1980, croissant plus vite que l'inflation. Dans ce domaine aussi, ce fut Harvard qui lança le mouvement, et ce dès 1978. Elle fut très vite suivie par ses consœurs de l'Ivy League. Néanmoins, les universités publiques n'adoptèrent la même politique qu'à la fin des années 1980 . Voulant rattraper leur retard, elles dépassèrent finalement leurs homologues privées dans les années 1990 et 2000 (Clayton M., 2003). Aujourd'hui, des droits d'inscription élevés sont devenus la norme dans toutes les universités privées et publiques selon la politique «à droit d'inscription élevé, aide élevée ».

21 Encouragée par le gouvernement depuis la fin des années 1980, cette politique a fermé l'accès des grandes universités publiques aux étudiants les plus pauvres, ceux-ci redoutant l'augmentation des frais de scolarité et craignant qu'elle ne soit pas 
compensée par un ajustement des bourses. Cet état d'esprit, de manière paradoxale, a avantagé les universités privées qui sont passées maîtres dans ce que l'on appelle «le jeu de l'aide aux étudiants » (McPherson M., Schapiro M., 1998). Encouragées par leur succès à attirer les enfants de milieux modestes et sous la pression de l'opinion publique et $\mathrm{du}$ Congrès, réagissant aux profits faramineux réalisés sur leurs endowments, les universités d'élite privées ont mis en place ces dernières années une politique de "non prêt» (no-loan policy) pour les étudiants de milieux modestes avec force publicité et sans s'interroger sur ce que cela allait coûter ${ }^{17}$.

La crise a donc frappé des universités en pleine fièvre spéculative, vivant au-dessus de leurs moyens dans une course éperdue au « toujours plus » et ayant perdu tout sens de la modération dans leurs objectifs.

\section{Les conséquences de la crise de 2008-2009 sur le fonctionnement des universités : le dur retour à la réalité et le renforcement de la compétition}

23 La première conséquence de la crise de 2008-2009 sur l'enseignement supérieur, la plus médiatisée même si elle ne concerne qu'un petit nombre d'institutions, a été la chute spectaculaire des endowments des grandes universités d'élite privées. Harvard et Yale furent particulièrement touchées. Entre 2008 et 2009 l'endowment d'Harvard a perdu presque $30 \%$ de sa valeur mettant l'université dans la pire situation financière qu'elle ait connue dans son existence ${ }^{18}$. Yale, qui mit un certain temps à dévoiler ses pertes, avoua en fin de compte ne pas avoir fait mieux qu'Harvard ${ }^{19}$. Même si David Swensen ne renia pas ses choix (Freeland, C., 2009), la gestion des endowments conduite par les universités d'élite, qui jusque-là avait servi de cas d'école, fut sévèrement remise en cause. On s'interrogea sur cette politique du «toujours plus» qui avait poussé les gestionnaires des endowments à emprunter pour pouvoir placer encore plus d'argent et qui avait laissé les universités sans aucune liquidité pour faire face à la crise, les obligeant ainsi à vendre au plus mauvais moment (Condon B., Vardi N., 2009). On se demanda comment ces gestionnaires si compétents et autant payés avaient pu faire perdre en quelques mois aux quatre grandes universités de l'Ivy League (Harvard, Yale, Princeton et Stanford) une somme d'argent supérieure à la valeur des endowments de toutes les autres institutions d'enseignement supérieur américaines (Rosenfield A., 2009).

Même si certaines tirèrent mieux leur épingle du jeu que d'autres, toutes les universités virent leurs réserves financières fondre comme neige au soleil (Blumenstyk G., 2009). Les revenus de l'endowment représentant pour beaucoup d'universités privées plus du tiers de leur budget de fonctionnement ${ }^{20}$, leur effondrement entraîna une réduction drastique des coûts à tous les niveaux. À Harvard, le budget de la Faculté des Arts et des Sciences fut ainsi réduit en 2009-2010 de 20 \%, entraînant des suppressions de postes et de cours.

Dans le cadre de ces restrictions budgétaires, certains programmes d'aide, comme par exemple la politique du "non prêt», furent remis en cause. Les bénéfices spectaculaires des années précédentes et la pression populaire avaient poussé les grandes universités privées non seulement à aider financièrement leurs étudiants à couvrir leurs frais de scolarité, notamment en transformant leurs prêts en bourses, mais les avaient incitées à une largesse extrême ${ }^{21}$. Harvard, comme d'habitude, avait 
montré l'exemple. En 2004, le président d'Harvard, Larry Summers, avait étonné le monde de l'enseignement supérieur en déclarant que les études à Harvard seraient gratuites pour les étudiants dont les parents gagnaient moins de 40000 dollars par an (Basinger J., Smallwood S., 2004). Deux ans plus tard, ce chiffre passait à 60000 dollars (Heller D., 2007). En décembre 2007, quelques mois seulement avant l'effondrement du marché financier, Drew Faust, la nouvelle présidente d'Harvard, poursuivait la même politique en annonçant qu'Harvard ne demanderait qu'une contribution de $10 \%$ aux étudiants dont les parents avaient un revenu annuel inférieur à 180000 dollars (Hoover E., 2007 ; Farrell E., 2008). La crise remit en question cette politique généreuse.

Les universités privées ne furent pas les seules à revoir leur politique d'aide aux étudiants à cause de la crise. Les États fédérés reconsidérèrent de la même façon les programmes d'avancement des frais d'études (prepaid tuition plans) qu'ils avaient mis en place dans les années 1990. Ces dispositifs, qui permettaient aux familles de payer tout ou partie des frais de scolarité futurs de leurs enfants aux prix d'aujourd'hui, reposaient sur l'idée que la rentabilité de l'argent placé serait supérieure à l'augmentation des frais de scolarité. Or, la crise financière a entraîné l'effondrement des actifs de ces plans alors que les frais de scolarité ont continué à grimper. Les États, eux-mêmes dans une situation économique très difficile, n'ont pas eu les moyens de combler la différence (Carey K., 2009). La situation est d'autant plus compliquée que la crise, de manière paradoxale, a accru la popularité de ce système auprès des familles qui voient là un moyen de se protéger contre des hausses encore plus importantes des frais de scolarité en bloquant leur argent pour s'assurer que leurs enfants puissent aller à l'université. Cet état de fait a poussé certains États, comme le Texas, à transmettre le poids des pertes éventuelles de ces plans aux universités, voyant là une façon non seulement de se dégager des engagements financiers qu'ils ne peuvent plus assumer, mais aussi un moyen d'empêcher les universités de trop augmenter leurs frais de scolarité. De nouveau, les États se déchargent sur les institutions d'enseignement supérieur sans se poser la question de savoir comment elles vont pouvoir gérer ces frais supplémentaires en cette période de crise.

Pour répondre à ces nouvelles contraintes financières, toutes les universités ne sont pas armées de la même manière et à la sortie de cette crise il y aura peut-être des gagnants mais il y aura aussi sûrement des perdants. Les riches universités privées ainsi que les grandes universités publiques ont plus de chance d'être du côté des vainqueurs. Par contre les petites universités privées ainsi que les universités publiques qui se trouvent dans des États fortement touchés par la crise vont nécessairement avoir plus de mal à s'en sortir'22.

\section{Conclusion : la résilience des grandes universités américaines}

Même si ce sujet n'appelle pas vraiment de conclusion tant il reste ouvert et incertain, on peut néanmoins s'interroger sur l'évolution du rôle de l'État dans l'enseignement supérieur américain et, plus généralement, dans la société américaine. Pendant plus de quarante ans, en effet, le discours politique dominant a présenté l'État, et notamment l'État fédéral, comme une gêne pour le bon fonctionnement de la société plutôt que comme une solution. Pendant plus de quarante ans aussi, le système d'enseignement supérieur s'est privatisé, adoptant les mêmes stratégies de gestion que les entreprises 
privées et leur donnant même parfois l'exemple dans la modernisation et la complexification de leurs instruments financiers. La recherche et l'accumulation de ressources financières ont pris le pas sur la réflexion pédagogique. Les présidents des universités sont devenus des collecteurs de fonds à plein temps, reléguant au second rang leur mission universitaire. Même si quelques voix se sont fait entendre pour avertir du danger de cette politique du « tout privé » et du règne de la rentabilité, elles ont été noyées dans le flot des discours des défenseurs de « l'argent roi ».

Après avoir été "le problème ", l'État fédéral, devant l'ampleur de la crise, apparait aujourd'hui, de nouveau, et pour beaucoup, comme la solution. Peu de voix se sont en effet fait entendre dans l'enseignement supérieur pour critiquer le plan de relance du président Obama de 2009 (The American Recovery and Reinvestment Act of 2009). Les 50 milliards promis aux États fédérés pour les aider à faire face à leurs difficultés budgétaires ont été perçus comme un moyen pour amortir l'impact de la crise sur les universités publiques. De la même façon, les enseignants-chercheurs se sont précipités pour présenter des dossiers afin de bénéficier d'une partie des 10 milliards offerts sous la forme de bourses de recherche par le National Institute of Health dans le cadre de ce programme de soutien fédéral (Basken P., 2009).

Ce plan a pourtant des limites. Même s'il ne faut pas sous-estimer son impact et le rôle essentiel de l'État fédéral dans le financement de l'enseignement supérieur, notamment au niveau de la recherche et de l'octroi de bourses aux étudiants, c'est l'apport supplémentaire de capitaux privés qui a été dans cette crise, comme dans beaucoup de crises précédentes, le facteur primordial de redressement des grandes universités. En effet, seulement deux ans après la crise, les endowments des universités ont recommencé à croître de manière spectaculaire. En 2010, l'endowment d'Harvard a augmenté de $21 \%$ et celui de Yale de $22 \%$ portant leurs valeurs respectives à 30 et 20 milliards de dollars et effaçant ainsi, en à peine deux ans, les pertes subies préalablement. Cette remontée impressionnante des endowments est aussi vraie pour les autres grandes universités, privées aussi bien que publiques. Les excellents résultats des universités sont dus à la bonne reprise des marchés financiers mais aussi à la mobilisation exceptionnelle des anciens élèves. Yale a clos en 2011 une campagne de levée de fonds sur 7 ans dont le montant initial avait été fixé à 3 milliards de dollars et qui a été porté en 2008 à 3,5 milliards pour aider l'université à faire face aux conséquences de la crise ${ }^{23}$. La même année, les donations faites à Harvard ont augmenté de $7 \%$ par rapport à l'année précédente atteignant un record de 639 millions $^{24}$. Ce record fut battu l'année suivante par Stanford qui a réussi à collecter pour la première fois dans l'histoire de l'enseignement américain plus d'un milliard de dollars en à peine un an (Lewin T., 2013). Même si elles ne figurent pas aux premiers rangs, les grandes universités publiques ne sont pas loin dans cette course aux records de collectes de fonds (Biemiller L., 2012).

31 Cette résilience des universités d'élite aux crises n'est pas nouvelle. Harvard a célébré en 2011 le $375^{\text {ème }}$ anniversaire de sa fondation, Yale le $310^{\text {ème }}$ et Stanford, qui fait figure de benjamine à côté de ses deux aînées, ses 120 années d'existence. Leur survie au cours des siècles est due à cette faculté de mobiliser en temps de crise non seulement le soutien public mais aussi et surtout le soutien privé. On peut donc raisonnablement penser que l'argent privé restera encore, dans les années à venir, une ressource prépondérante pour la majorité des universités. Et sans aller aussi loin que James Duderstadt - ancien président de l'université du Michigan et membre de la Commission 
sur le Futur de l'Enseignement Supérieur mise en place par le Ministère de l'Éducation en 2006 - qui considérait que l'enseignement supérieur américain était devenu «un système intensément darwinien dans lequel les institutions les plus fortes et les plus riches sont devenues des prédateurs $\aleph^{25}$, il semble que la compétition très âpre dans la recherche de fonds demeure plus que jamais une condition essentielle de survie pour les universités américaines et le deviendra aussi, sans doute, dans les années à venir pour les universités européennes en raison de l'évolution prévisible de la situation économique et politique.

\section{BIBLIOGRAPHIE}

Basinger Julianne, Smallwood Scott, « Harvard gives a break to parents who earn less than \$ 40,000 a year ", The Chronicle of Higher Education, March 12, 2004. Disponible en ligne : http:// chronicle.com/article/Harvard-Gives-a-Break-to/33465/. Page consultée le 21 juin 2013.

Basken, Paul, « NIH is deluged with 21000 grant applications for Stimulus Funds », The Chronicle of Higher Education, June 9, 2009. Disponible en ligne : http://chronicle.com/article/NIH-IsDeluged-With-21000/47306/. Page consultée le 21 juin 2013.

Biemiller, Lawrence, « Gifts to Colleges rose 8,2\% in 2011, survey finds », The Chronicle of Higher Education, February 15, 2012. Disponible en ligne : http://chronicle.com/article/Gifts-to-CollegesRose-82-in/130786/. Page consultée le 21 juin 2013.

Blumenstyk, Goldie, « Market collapse weighs heavily on endowments », The Chronicle of Higher Education, February 6, 2009. Disponible en ligne : http://chronicle.com/article/Market-CollapseWeighs-Heavily/1481. Page consultée le 21 juin 2013.

Bok, Derek, Universities in the marketplace: the commercialization of higher education, Princeton, Princeton University Press, 2003.

Breneman David, "For Colleges, this is not just another recession », The Chronicle of Higher Education, June 4, 2002. Disponible en ligne : http://chronicle.com/article/For-Colleges-This-IsNot-Just/27351/. Page consultée le 21 juin 2013.

Carey, Kevin, « College Savings Plans: a bad gamble », The Chronicle of Higher Education, May 8, 2009. Disponible en ligne : http://chronicle.com/article/College-Savings-Plans-a-Bad/44310. Page consultée le 21 juin 2013.

Carlson, Scott, « Moody's sees stiff challenges for Colleges - especially private ones - in next year », The Chronicle of Higher Education, January 6, 2009. Disponible en ligne : http:// chronicle.com/article/Moodys-Sees-Stiff-Challenges/1432. Page consultée le 21 juin 2013.

Center for the Study of Education Policy, Recession, Retrenchment, and Recovery: State higher education funding and student financial aid, Lumina Foundation for Education, October 2006. Chronicle of Higher Education, «Colleges and universities endowments, 2006-2007 », The Chronicle Almanac, 2008. Disponible en ligne : http://chronicle.com/premium/stats/endowments/ results.php?year=2008. Page consultée le 21 juin 2013. 
Clayton, Mark, « Tuitions up steeply nationwide », Christian Science Monitor, August 11, 2003. Disponible en ligne, http://www.csmonitor.com/2003/0811/p01s03-ussc.html. Page consultée le 21 juin 2013.

Coldren, Sharon, The Constant quest: raising billions through capital campaigns, Washington D.C., American Council on Education, 1982.

Commission on the Future of Higher Education, A test of leadership: charting the future of higher education, A report of the Commission appointed by Secretary of Education Margaret Spellings, U.S. Department of Education, 2006. Disponible en ligne : http://www.ed.gov/about/bdscomm/ list/hiedfuture/index.html. Page consultée le 21 juin 2013.

Condon, Bernard, Vardi, Nathan, « How Harvard's Investing Superstars crashed: when genius fails, it fails big ", Forbes, February 20, 2009. Disponible sur http://www.forbes.com/2009/02/20/ harvard-endowment-failed-business_harvard.html. Page consultée le 21 juin 2013.

Conley, Aaron, Tempel, Eugene, « Philanthropy » in Douglas Priest, Edward St John, Privatization and public universities, Bloomington, Indiana University Press, 2006, p. 151-72.

Cronin, Joseph, Horton, Howard, « Will higher education be the next bubble to burst? », The Chronicle of Higher Education, May 22, 2009. Disponible en ligne, http://chronicle.com/article/WillHigher-Education-Be-the/44400/. Page consultée le 21 juin 2013.

Couturier, Lara, Checks and balances at work: The restructuring of Virginia's public higher education system, The National Center for Public Policy and Higher Education, National Center Report 06-3, June 2006. Disponible en ligne : http://www.highereducation.org/catreports/ governance_state_policy_leadership.shtml. Page consultée le 20 septembre 2013.

Erhenberg, Ronald, « The perfect storm and the privatization of Public Higher Education », Change, vol. 38, $\mathrm{n}^{\circ} 1$, January/February 2006. Disponible en ligne : http://

digitalcommons.ilr.cornell.edu/workingpapers/. Page consultée le 21 juin 2013.

Ehrenberg, Ronald, What's happening to public higher education: the shifting financial burden, Baltimore, The Johns Hopkins University Press, 2006.

Farrell, Elizabeth, « The changing face of student aid », The Chronicle of Higher Education, April 4, 2008. Disponible en ligne : http://chronicle.com/article/The-Changing-Face-of-Student/34821/. Page consultée le 21 juin 2013.

Freeland, Chrystia, « Lunch with David Swensen », Financial Times, October 8, 2009. Disponible en ligne : http://www.ft.com/cms/s/0/dd91a3ec-b461-11de-

bec8-00144feab49a.html\#axzz2X4OusuyL. Page consultée le 21 juin 2013.

Gladieux, Lawrence, Wolanin, Thomas, « A charter for federal policy towards postsecondary education: the education amendments of 1972 ", Journal of Law and Education 4, April 1975, p. 301-324.

Gose, Ben, « Changes at Elite Colleges Fuel Intense Competition in Student Aid », The Chronicle of Higher Education, February 5, 1999. Disponible en ligne : http://chronicle.com/article/Changes-atElite-Colleges-Fuel/21202/. Page consultée le 21 juin 2013.

Harvard Business School, « Yale University Investments Office: July 2000 », Harvard Business School Case No 9-201-129, Boston, Harvard Business School Publishing, 9-201-129, March 4, 2001.

Harvard Business School, Harvard Management Company, Boston, Harvard Business School Publishing, 9-201-129, October 23, 2001. 
Harvard Magazine, « Yale capitulates », Harvard Publishing, September 11, 2009. Disponible en ligne : HTTP://HARVARDMAGAZINE.COM/BREAKING-NEWS/YALE-ENDOWMENT-LOSSES-SPENDING-CUTS. Page consultée le 20 septembre 2013.

Harvard University Financial Report, Fiscal Year 2009, Fiscal Year 2011.

Hebel, Sara, «States start to consider the idea of charter colleges ", The Chronicle of Higher Education, March 24, 2000. Disponible en ligne : http://chronicle.com/article/States-Start-toConsider-the/15058. Page consultée le 21 juin 2013.

Hebel, Sara, « Public Colleges feel impact of the economic downturn », The Chronicle of Higher Education, July 20, 2001. Disponible en ligne : http://chronicle.com/article/Public-Colleges-FeelImpact-of/11993/. Page consultée le 21 juin 2013.

Hebel, Sara, « As term limits kick in, State colleges fear the impact », The Chronicle of Higher Education, September 28, 2001. Disponible en ligne : http://chronicle.com/article/As-TermLimits-Kick-In-State/31012/. Page consultée le 21 juin 2013.

Heller, Donald, « How Harvard foils its own good intentions », The Chronicle of Higher Education, December 17, 2007. Disponible en ligne : http://chronicle.com/article/How-Harvard-Foils-ItsOwn-Good/123526/. Page consultée le 21 juin 2013.

Hoover Eric, « Harvard's new aid policy raises the stakes », The Chronicle of Higher Education, December 21, 2007. Disponible en ligne : http://chronicle.com/article/Harvards-New-Aid-Policy/ 5175/. Page consultée le 21 juin 2013.

Kane, Thomas, Orszag, Peter, « Higher education spending: the role of Medicaid and the business cycle », The Brookings Institution Policy Brief\#124, September 2003.

Kelderman, Eric, « Colleges press new ideas as they brace for bumpy State-budget sessions », The Chronicle of Higher Education, January 9, 2009. Disponible en ligne : http://chronicle.com/article/ Colleges-Press-New-Ideas-as/33330/. Page consultée le 21 juin 2013.

Leubsdorf, Ben, « The problem with term limits », The Chronicle of Higher Education, July 28, 2006. Disponible en ligne : http://chronicle.com/article/The-Problem-With-Term-Limits/10292. Page consultée le 21 juin 2013.

Lewin, Tamar, « Report Says Stanford is first university to raise \$ 1 billion in a single year », The New York Times, February 20, 2013. Disponible en ligne : www.nytimes.com/2013/02/21/ education/stanfords-fund-raising-topped-1-billion-in-2012.html ?_r =0. Page consultée le 21 juin 2013.

Lord, Mimi, 2005 NACUBO Endowment Study: Highlights and Trends, TIAA-CREF Institute, February 2006. Disponible en ligne : http://www.nacubo.org/Search_Results_Page.html? q=2005\%20Endowment\%20study. Page consultée le 21 juin 2013.

Lorin, Janet, « Endowment losses threaten no-loan policies », Bloomberg, August 25, 2009.

Disponible en ligne : http://www.bloomberg.com/apps/news?pid=20603037\&sid=alEzZV_OZ9xk.. Page consultée le 21 juin 2013.

Lyall, Katherine, Sell, Kathleen, The true genius of America at risk: Are we losing our public universities to de facto privatization? American Council on Education, Praeger, 2006.

Masseys-Bertonèche, Carole, Philanthropie et grandes universités privées : pouvoir et réseaux d'influence, Bordeaux, PUB, 2006. 
McGuinness, Aims, " The States and higher education », in Philip Altbach, Robert Berdahl, Patricia Gumport, American higher education in the 21st century, Baltimore, The Johns Hopkins University Press, 1999, p. 183-215.

McLendon, Michael, Mokher, Christine, « The origins and growth of state policies that privatize higher education ", in Christopher Morphew, Peter Eckel, Privatizing the public university: perspectives from across the Academy, The Johns Hopkins University Press, 2009, p. 15-17.

McPherson, Michael, Schapiro, Morton, The student aid game: meeting needs and rewarding talent in American higher education, Princeton, Princeton University Press, 1998.

National Center for Education Statistics, Digest of Education Statistics, U.S. Department of Education, Institute of Education Sciences, National Center for Education Statistics, 1976.

Okunade, Albert, « What factors influence State appropriations for public higher education in the United States? » Journal of Education Finance, Fall 2004, p. 123-138.

Parsons, Michael, Power and politics: federal higher education policymaking in the 1990s, New York, State University of New York Press, 1997.

Richburg, Keith, Vick, Karl, « Fiscal crisis is hitting some states hard », Washington Post, October 4, 2008. Disponible en ligne : http://articles.washingtonpost.com/2008-10-04/news/ 36892620_1_bond-sale-municipal-bonds-matt-fabian. Page consultée le 21 juin 2013.

Rosenfield, Andrew, « Understanding Endowment », Forbes, March 4, 2009. Disponible en ligne : http://www.forbes.com/2009/03/03/harvard-university-investment-opinionscontributors_endowment.html. Page consultée le 21 juin 2013.

Rubenstein, Robert, Helping oustanding pupils educationally: public policy issues of the Georgia HOPE scholarship program and the lottery for education, The policy Brief Series, Center for Policy Research at the Maxwell School of Syracuse University, 2003. Disponible en ligne : http://wwwcpr.maxwell.syr.edu. Page consultée le 21 juin 2013.

Schmidt, Peter, « Prepaid-tuitions plans: popularity and peril », The Chronicle of Higher Education, March 24, 2000. Disponible en ligne : http://chronicle.com/article/Prepaid-Tuition-Plans-/ 10086/. Page consultée le 21 juin 2013.

Schmidt, Peter, « Weakening the grip of multicampus boards ", The Chronicle of Higher Education, March 23, 2001. Disponible en ligne : http://chronicle.com/article/Weakening-the-Grip-of/ 14279/. Page consultée le 21 juin 2013.

Schmidt, Peter, « Revamping of Education Governance in Florida Reveals a New Political Order », The Chronicle of Higher Education, May 17, 2002, p. A32. Disponible en ligne : http://chronicle.com/ article/Revamping-of-Education/27369/. Page consultée le 21 juin 2013.

Selingo, Jeffrey, « The disappearing State in higher education », The Chronicle of Higher Education, February 2003. Disponible en ligne $:$ http://chronicle.com/article/The-Disappearing-State-in/ 21186/. Page consultée le 21 juin 2013.

Swensen, David, Pioneering Porfolio Management, New York, Free Press, 2000.

Yale University financial Report, 2008-2009, 2010-2011.

Zemsky, Robert, « Have we lost the 'Public' in higher education », The Chronicle of Higher Education, May 30, 2003. Disponible en ligne : http://chronicle.com/article/Have-We-Lost-the-Public-in/ 21529/ Page consultée le 21 juin 2013. 


\section{NOTES}

1. Deux économistes, Thomas Kane et Peter Orszag, montrent que les choix budgétaires difficiles que les États ont dû faire pour répondre aux nouvelles charges et, notamment, à l'augmentation des frais de couverture de soins du programme Medicaid ont été effectués au détriment du financement des universités publiques. Voir Kane \& Orszag (2003).

2. Le National Bureau of Economic Research qui détermine les périodes de récessions économiques a officialisé quatre crises entre 1979 et 2004, deux au début des années 1980, une au début de 1990 et celle de 2001. Voir Center for the Study of Education Policy (2006).

3. Entre 1980 et 2003, la proportion de l'argent des États qui a été attribuée à l'enseignement supérieur public est passée de $44 \%$ à $32 \%$. Voir Selingo (2003). Hebel (2001), Zemsky (2003).

4. Un des exemples le plus frappant du changement de gouvernance des universités américaines a été celui de l'université de Virginie. Voir Couturier (2006).

5. Le montant de l'argent investi dans ces programmes, Prepaid tuition and College Saving Plans, s'est accru en dix ans de plus de $3000 \%$ passant de 2,4 milliards de dollars en 1996 à 72,3 milliards en 2005. Voir McLendon \& Mokher (2009) ; Schmidt (2000).

6. Sur les conséquences du programme HOPE, voir Rubenstein (2003).

7. Voir Digest of Education Statistics, (1976 edition : 132).

8. Pour une analyse du financement des grandes universités privées à la fin des années 1960 et de son évolution après la crise pétrolière des années 1970, voir Masseys-Bertonèche (2006).

9. Nous avons gardé le terme anglo-saxon endowment dans la mesure où, comme les termes trust ou trustee, il n'a aucune équivalence en français. La traduction la plus proche du terme endowment qui serait, selon nous, "portefeuilles d'actifs non cessibles et soumis à conditions ", ne pourrait que gêner la lecture du fait de sa longueur.

10. En 2000, elle employait 185 personnes, dont 45 gérants de portefeuilles et avait un budget de fonctionnement de 42 millions de dollars. Voir Harvard Business School, Harvard Management Company, (2001).

11. Voir le livre qu'il a publié dans lequel il développe ses arguments justifiant l'investissement pour les universités de leur endowment dans le capital-risque et autres actifs non traditionnels. Swensen (2000).

12. Pendant les 20 dernières années, Yale a réalisé une rentabilité moyenne de $16,1 \%$ par an. Pour plus de détails sur la gestion de l'endowment de Yale, voir l'étude de cas réalisée par la Harvard Business School, « Yale University Investments Office : July 2000 » (2001).

13. La moyenne des taux de croissance de l'endowment d'Harvard entre 1995 et 2000 était de $20,5 \%$ alors que celle de Yale était de 19,5\% et, sur les dix ans, Harvard et Yale arrivaient presque à égalité avec un taux de croissance moyen de respectivement $15,3 \%$ et $15 \%$.

14. Pour une analyse de l'évolution des endowments et notamment des 56 institutions ayant des endowments dépassant 1 milliard de dollars, voir Lord (2006) et Chronicle of Higher Education (2008).

15. Ces universités publiques sont, par ordre de richesse: University of Texas System, University of Michigan, Texas A\&M University System, University of California, University of Virginia. Ibid.

16. Pour pouvoir recevoir des fonds privés, elles ont dû créer des organisations à but non lucratif qui, bien qu'apparemment indépendantes, ne servent qu'à assurer le transfert de fonds. Un des premiers exemples a été la Kansas University Endowment Association, établie en 1891 pour recevoir des donations des anciens élèves de l'université du Kansas, et la première collecte de fonds fut lancée par l'université Indiana en 1921, Voir Conley \& Tempel (2006).

17. Lancée avec force publicité par Princeton en 1998, cette politique consistant, pour les étudiants de milieu modeste, à substituer aux prêts personnels des bourses ou des exemptions de frais de scolarité a vite été suivie par l'ensemble des institutions d'élite. Voir Gose (1999).

18. Voir Harvard University Financial Report, Fiscal Year 2009. 
19. Voir Harvard Magazine, "Yale capitulates ", September 11, 2009. Disponible en ligne : http:// HARVARDMAGAZINE.COM/BREAKING-NEWS/YALE-ENDOWMENT-LOSSES-SPENDING-CUTS.

20. En 2008, 34,5\% des revenus d'Harvard et $37 \%$ des revenus de Yale provenaient des rentabilités sur investissement de leur endowment. Voir Harvard University Financial Report, Fiscal Year (2009) et Yale Financial Report, 2008-2009.

21. Les 16 premières universités classées dans le rapport de U.S. News de 2010, à l'exception de Johns Hopkins, ont adopté une variante de ce programme de remplacement des prêts par des bourses. Voir Lorin (2009). Disponible sur http://www.bloomberg.com/apps/news? pid=20603037\&sid=alEzZV_OZ9xk.

22. Sur le lien entre les finances des États fédérés et des universités qu'ils abritent, voir Kelderman (2009).

23. Voir Yale Financial Report, 2010-2011.

24. Voir Harvard University Financial Report, Fiscal Year 2011.

25. Voir Commission on the Future of Higher Education (2006).

\section{RÉSUMÉS}

L'article décrit l'impact de la crise financière de 2008-2009 sur la gestion des universités américaines. Il analyse, dans une première partie, les changements qu'a subis l'enseignement supérieur américain ces vingt dernières années pour s'adapter et répondre aux crises précédentes. Puis, dans une deuxième partie, il étudie comment cette évolution du fonctionnement des universités et de leurs modes de financement a accru leur vulnérabilité face à cette nouvelle crise. Il conclut en soulignant les facteurs de résilience des grandes universités et leurs facultés d'adaptation aux changements en période de crise.

This article focuses on the impact of the 2008-2009 financial crisis on the management of American universities. First, it analyses the changes that the American higher education system underwent in the last twenty years to adapt to previous economic crises. Then, it shows how the evolution of the sources of funding has made the universities more vulnerable in dealing with the 2008-2009 crisis. As a conclusion, it underlines the resilient factors of Top Research Universities and their adaptability to change in time of crisis.

El artículo describe el impacto de la crisis financiera de 2008 sobre la gestión de las universidades estadounidenses. En una primera parte, se analizan los cambios que la educación superior en los Estados Unidos ha experimentado durante los últimos veinte años para adaptarse a las anteriores crisis económicas. Luego, examina cómo estos cambios, tanto en el funcionamiento de las universidades como en sus métodos de financiación, las han vuelto más vulnerables para enfrentar la crisis actual. Se concluye destacando los factores de resiliencia de las grandes universidades estadounidenses y su capacidad de adaptación a los cambios en periodo de crisis. 


\section{INDEX}

Mots-clés : Crise financière, universités américaines, enseignement supérieur américain, sources de financement

Keywords : Financial crisis, American universities, American higher education system, university funding sources

Palabras claves : Crisis financiera, universidades de los EE.UU., sistema de educación superior norteamericano, fuentes de financiación

\section{AUTEUR}

\section{CAROLE MASSEYS-BERTONÈCHE}

Maître de Conférences à l'Université de Bordeaux, ses domaines de recherche sont le secteur non lucratif, la philanthropie et l'enseignement supérieur aux États-Unis. Elle travaille plus particulièrement sur le rôle du réseau philanthropique dans le fonctionnement des universités américaines.

Après avoir centré son travail de recherche sur les États-Unis, elle a évolué ces dernières années vers une vision comparatiste entre les États-Unis et l'Europe. Elle étudie depuis maintenant presque quatre ans les phénomènes d'influences croisées entre les États-Unis et l'Europe dans les réformes récentes de l'enseignement supérieur et elle a publié plusieurs articles et chapitres d'ouvrage sur ce thème.

Pour approfondir le sujet, elle a bénéficié, pour l'année 2012-2013, d'une invitation de l'Université Harvard en tant que Visiting Scholar. carole.bertonecheatu-bordeaux4pointfr 